\title{
SEDUM NEOVOLCANICUM (CRASSULACEAE) UNA ESPECIE NUEVA ORIGINARIA DEL CENTRO-OCCIDENTE DE MÉXICO
}

\author{
Emmanuel Pérez-Calix ${ }^{1}$ \\ Instituto de Ecología, A.C. \\ Centro Regional del Bajío \\ Apartado postal 386 \\ 61600 Pátzcuaro, Michoacán \\ $\mathrm{E}$ \\ Ignacio García Ruiz'2,3 \\ CIIDIR-IPN Michoacán \\ Justo Sierra 28 \\ 59510 Jiquilpan, Michoacán
}

\begin{abstract}
RESUMEN
Se describe y propone como especie nueva para la ciencia a Sedum neovolcanicum, con base en plantas originarias de los estados de Jalisco y Michoacán en la porción oeste de la región fisiográfica denominada Eje Neovolcánico (Eje Volcánico Transversal). El nuevo taxon se asemeja a Sedum bourgaei Hemsl., a Sedum tortuosum Hemsl. y a Sedum multiflorum R. T. Clausen en sus flores de color blanco o blanco-rosado; pero el hábito, el número de flores por inflorescencia, la inflorescencia sin ramificar y la longitud de los pétalos y sépalos distinguen a Sedum neovolcanicum de las otras entidades mencionadas.
\end{abstract}

Palabras clave: Crassulaceae, Sedum, México.

\section{ABSTRACT}

Sedum neovolcanicum is described as new species, based on plants collected in the western portion of the geographical region known as the Trans-Mexican Volcanic Belt, in the states of Jalisco and Michoacán. The new taxon resembles Sedum bourgaei Hemsl., S. tortuosum Hemsl. and S. multiflorum R. T. Clausen in possessing white or rosy-white flowers. However, it is distinguished from these species by its habit, number of flowers per inflorescence, unbranched inflorescence, and petals and sepals length.

Key words: Crassulaceae, Sedum, Mexico.

\footnotetext{
${ }^{1}$ Trabajo realizado con apoyo económico del Instituto de Ecología, A.C. (cuenta 902-03), del Consejo Nacional de Ciencia y Tecnología y de la Comisión Nacional para el Conocimiento y Uso de la Biodiversidad.

${ }^{2}$ Trabajo apoyado parcialmente por la Comisión Nacional para el Conocimiento y Uso de la Biodiversidad (Proyecto H-304).

${ }^{3}$ Becario de la COFAA del Instituto Politécnico Nacional.
} 
Durante las exploraciones ligadas al estudio florístico del Parque Nacional Pico del Tancítaro, en el centro-occidente del estado de Michoacán, se colectó una planta del género Sedum que no resultó posible identificar como alguna de las especies descritas en la literatura. Por otro lado, en la revisión del herbario del Instituto de Ecología, A.C., Centro Regional del Bajío (IEB) se observaron ejemplares originarios de los volcanes Nevado de Colima y de Fuego en el estado de Jalisco, los que sin duda pertenecen a la misma entidad taxonómica, pero que estaban erróneamente determinados. Acorde con lo anterior nos inclinamos por proponer como especie nueva para la ciencia a:

Sedum neovolcanicum Pérez-Calix et I. García sp. nov. Fig. 1.

Planta herbacea perennis epiphytica vel rupestris, pendens vel decumbens, glabra, papillata in partibus junioribus, usque $40 \mathrm{~cm}$ longa; caules ad basim $5 \mathrm{~mm}$ diametro, ramosi; folia spiraliter disposita, sessilia, laminis lineari-oblanceolatis, (6)10-22 mm longis, 1.5-3 mm latis, papillatis; flores solitarii vel in cincinnis usque 5 -floris ordinati, sessiles vel subsessiles, albi vel albo-rosei; calyx 5 sepalis inaequalibus, 5-13 mm longis, 2-3.5 mm latis; corolla ca. $1.5 \mathrm{~cm}$ diametro, petalis 5 liberis oblongo-ellipticis, $8-11 \mathrm{~mm}$ longis, $5 \mathrm{~mm}$ latis; nectaria ca. $0.7 \mathrm{~mm}$ longa atrorubra; follicula $7-8 \mathrm{~mm}$ longa.

Planta herbácea perenne, suculenta, epífita o rupícola, colgante o decumbente, glabra, papilosa en las partes tiernas, hasta de $40 \mathrm{~cm}$ de largo, profusamente ramificada desde la base; las plantas maduras con raíces tuberosas, fasciculadas; tallos de hasta $\pm 5 \mathrm{~mm}$ de diámetro en la base, inicialmente de color verde, grises en la madurez, con tonos rojizos en la porción media, corteza ligeramente reticulada en las partes maduras, algo exfoliante, con raíces adventicias en los nodos; hojas dispuestas en espiral, sésiles, linearoblanceoladas, de (6)10 a $22 \mathrm{~mm}$ de largo, 1.5 a $3 \mathrm{~mm}$ de ancho y de 1 a $1.5 \mathrm{~mm}$ de grueso, ápice redondeado, base brevemente espolonada, papilosas; flores solitarias 0 dispuestas en cincinos con hasta 5 flores, éstas sésiles o sobre pedicelos de 1 a $2.4 \mathrm{~mm}$ de largo; cáliz de 5 sépalos desiguales a subiguales, libres, suculentos, linear-oblongos a oblongos, de 5 a $13 \mathrm{~mm}$ de largo y de 2 a $3.5 \mathrm{~mm}$ de ancho, base ligeramente espolonada, de color verde, papilosos; corola de $1.5 \mathrm{~cm}$ de diámetro, pétalos 5, libres, oblongo-elípticos a elípticos, de 8 a $11 \mathrm{~mm}$ de largo y $5 \mathrm{~mm}$ de ancho, aquillados dorsalmente, ápice mucronado, de color blanco, blanco-rosado o blanco-verdoso, rosados dorsalmente cerca del ápice; estambres 10, 5 antepétalos de 6 a $9 \mathrm{~mm}$ de largo y 5 antesépalos de 7 a $8 \mathrm{~mm}$ de largo; nectarios de 0.6 a $0.8 \mathrm{~mm}$ de largo y ca. $0.4 \mathrm{~mm}$ de ancho, de color rojo oscuro; ovario formado por 5 carpelos fusionados en la base, de 6 a $8 \mathrm{~mm}$ de alto, estilos de $2 \mathrm{~mm}$ de largo; frutos en forma de folículos de 7 a $8 \mathrm{~mm}$ de largo, semillas numerosas.

TIPO: México, Michoacán, 27 km al W de Uruapan en línea recta: Barranca del Agua, 5.7 km al NE de Tancítaro, municipio de Tancítaro. Alt. 2660 m. Lat. 19²3'15" N; Long. 102020'22" W. Ladera sombreada cerca de un arroyo, bosque de coníferas (Pinus pseudostrobus - Abies religiosa), 14.XI.1998. I. García y G. Hernández 5542 (holotipo: IEB; isotipos por distribuirse a: CIMI, ENCB y MEXU).

Material adicional examinado: Michoacán: Cerro Tancítaro, $27 \mathrm{~km}$ al W de Uruapan en línea recta, al S-SE de la Barranca Rodada, municipio de Tancítaro, I. García, J. A. 
Pérez-Calix et al.: Sedum neovolcanicum Una Especie Nueva del Centro-Occidente de México

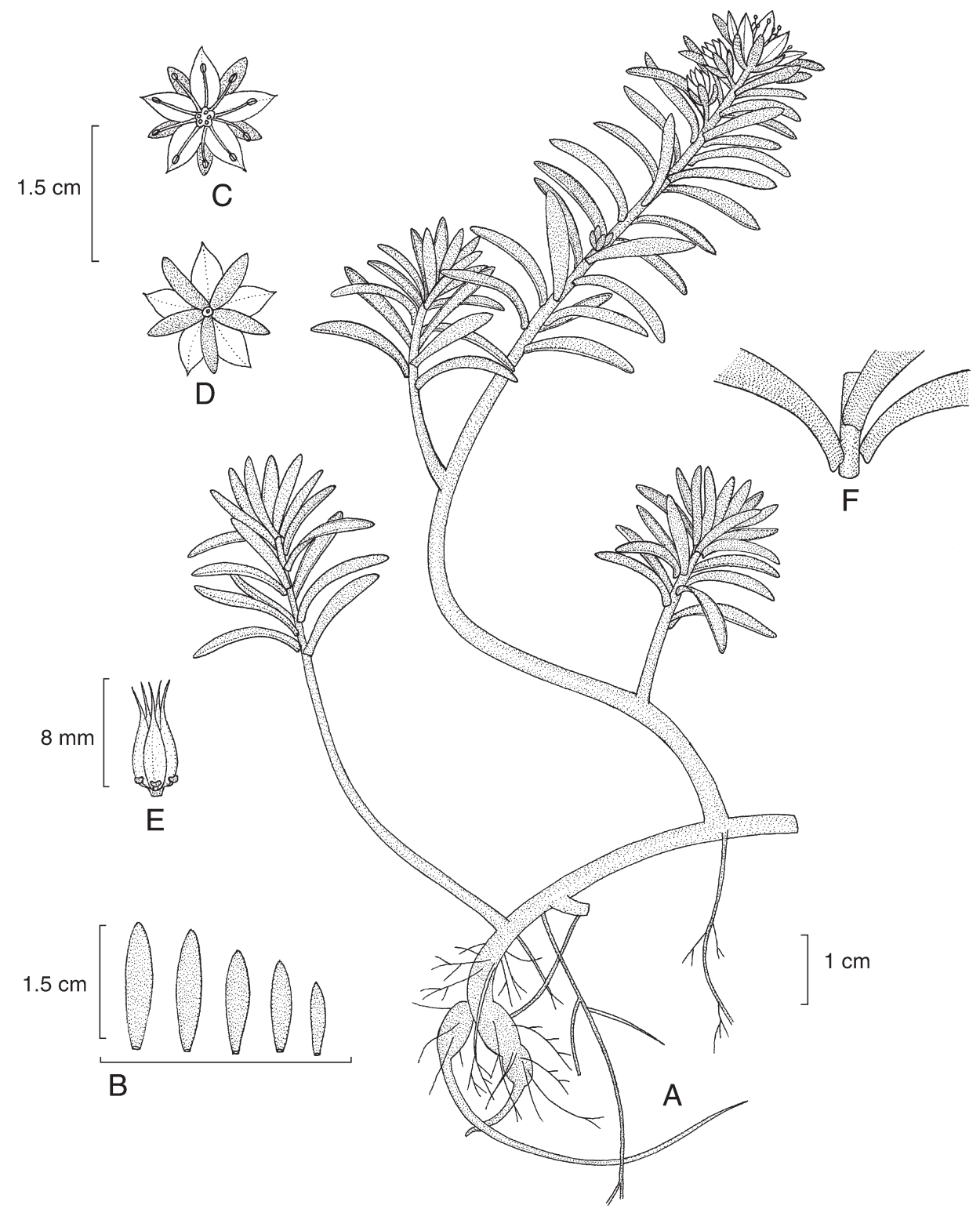

Fig. 1. Sedum neovolcanicum Pérez-Calix et I. García. A. Hábito de la planta; B. Variación de las hojas; C. Flor en vista ventral; D. Flor en vista dorsal; E. Gineceo y nectarios; F. Acercamiento de las bases de las hojas. Ilustrado por Rogelio Cárdenas Soriano. 
Machuca y M. Cházaro 4461 (CIMI, ENCB, IEB, MEXU, MICH, XAL); Cerro Tancítaro, $27 \mathrm{~km}$ al W de Uruapan en línea recta, aproximadamente $1 \mathrm{~km}$ al W del Cerro de la Cruz, municipio de Tancítaro (Lat. 19²3'55" N; Long. 102²0'40" W), I. García, J. A. Machuca y R. Acevedo 4660 (CIMI, IEB); Cerro de la Cruz, acceso por el Jazmín, municipio de Tancítaro, J. A. Machuca 8150 (CIMI, IEB); 8151 (CIMI, IEB). Jalisco: brecha al W de Atenquique, Volcán del Fuego, municipio de Zapoltitic, J. A. Machuca y M. Cházaro 6519 (IEB); Nevado de Colima, antes de llegar al refugio de Las Joyas, M. Cházaro, R. Ramírez y J. A. Pérez de la Rosa 4449 (IEB); Nevado de Colima, C. L. Díaz-Luna 18240 (GUADA, IEB); Nevado de Colima, H. H. Iltis et al. 33124 (IEB); Nevado de Colima, paraje Los Lioncitos, A. García y M. Zavala s/n (IEB).

Fenología, distribución y hábitat: Sedum neovolcanicum se ha encontrado en floración de mediados de septiembre a febrero. Es una planta rupícola, a veces epífita, entonces crece principalmente sobre Alnus jorullensis. Se ha colectado en cañadas húmedas y sombreadas, particularmente en laderas de exposición norte y noroeste con bosque de Pinus-Abies. Crece acompañada de Heuchera sp., Valeriana robertianifolia, Gnaphalium oxyphyllum, Lycopodium sp. y diversos helechos. Se conocen únicamente tres poblaciones que se sitúan en la porción occidental del Eje Neovolcánico, en altitudes de 2650 a 3250 m. El epíteto específico obedece a su distribución geográfica.

El género Sedum ha encontrado en la provincia fisiográfica conocida como Eje Neovolcánico las condiciones ambientales apropiadas para su establecimiento y diversificación. Así, Clausen (1959) registró 28 entidades específicas para esta unidad fisiográfica y posteriormente describió una más (Clausen, 1978). De acuerdo con este autor, son 16 los taxa endémicos a alguna porción de tal sistema montañoso. Adicionando la nueva entidad, se eleva a 30 el número de especies y a 17 el de endemismos registrados.

En la zona aledaña a los lugares en que crece Sedum neovolcanicum se han registrado 13 especies del género (Clausen 1959, 1978), de las que tres (Sedum tortuosum Hemsl., Sedum bourgaei Hemsl. y Sedum multiflorum R. T. Clausen) se asemejan en el color de la flor al nuevo taxon. Sin embargo, Sedum neovolcanicum tiene sépalos y pétalos de mayor longitud, pedicelos menores de $2.4 \mathrm{~mm}$ de largo o ausentes, flores solitarias o hasta cinco agrupadas en cincinos simples, mientras que las otras especies presentan una inflorescencia en forma de cima compuesta y un número mayor de flores por cincino. En particular de Sedum tortuosum se diferencia en que este último presenta hojas oblanceoladas a elíptico-espatuladas, de 4 a $11 \mathrm{~mm}$ de ancho, pedicelos florales de más de $2.4 \mathrm{~mm}$, así como en el nectario que es de color amarillo pálido, mientras que las hojas de $S$. neovolcanicum son linear-oblanceoladas, de 1.5 a $3 \mathrm{~mm}$ de ancho, pedicelos florales nulos o menores de $2.4 \mathrm{~mm}$ y el nectario es de color rojo oscuro. De Sedum bourgaei se separa porque el hábito de la nueva especie es decumbente o colgante, mientras que en esta última es generalmente erecto, tiene las hojas lineares y los pedicelos florales de mayor longitud. De S. multiflorum, se diferencia principalmente por su inflorescencia, ya que la de esta especie es una cima compuesta de 2 o más cincinos, mientras que la de $S$. neovolcanicum es una cima monocásica, además esta última presenta sépalos y pétalos mayores (Cuadro 1). 
Pérez-Calix et al.: Sedum neovolcanicum Una Especie Nueva del Centro-Occidente de México

Por otro lado, a Sedum neovolcanicum no se le ha podido relacionar con especies de otras regiones fisiográficas.

Cuadro 1. Comparación de Sedum neovolcanicum con especies relacionadas.

\begin{tabular}{|c|c|c|c|c|}
\hline & S. neovolcanicum & S. tortuosum & S. multiflorum & S. bourgaei \\
\hline Forma biológica & herbácea perenne & subfrútice & herbácea perenne & subfrútice \\
\hline $\begin{array}{l}\text { Tallo: } \\
\text { diámetro en } \\
\text { la base }(\mathrm{mm}) \\
\text { largo }(\mathrm{cm})\end{array}$ & $\begin{array}{l}5 \\
40 \text { ó menos }\end{array}$ & $\begin{array}{l}2 \text { a } 5 \\
25\end{array}$ & $\begin{array}{l}2 \text { a } 4 \\
9 \text { a } 26\end{array}$ & $\begin{array}{l}1 \text { a } 4 \\
15 \text { a } 60\end{array}$ \\
\hline $\begin{array}{l}\text { Hoja: } \\
\text { forma } \\
\text { largo }(\mathrm{mm}) \\
\text { ancho }(\mathrm{mm})\end{array}$ & $\begin{array}{l}\text { linear-oblanceolada } \\
\text { (6) } 10 \text { a } 22 \\
1.5 \text { a } 3\end{array}$ & $\begin{array}{l}\text { oblanceolada a } \\
\text { elíptico-espatulada } \\
9 \text { a } 27 \\
4 \text { a } 11\end{array}$ & $\begin{array}{l}\text { linear-oblanceolada } \\
11 \text { a } 16.7 \\
\pm 3\end{array}$ & $\begin{array}{l}\text { linear } \\
5 \text { a } 23 \\
1 \text { a } 3\end{array}$ \\
\hline $\begin{array}{l}\text { Inflorescencia: } \\
\text { tipo }\end{array}$ & cincino & $\begin{array}{l}\text { cima compuesta de } \\
2 \text { o más cincinos }\end{array}$ & $\begin{array}{l}\text { cima compuesta de } \\
\text { múltiples cincinos }\end{array}$ & $\begin{array}{l}\text { cima compuesta } \\
\text { de } 1 \text { a } 7 \text { cincinos }\end{array}$ \\
\hline $\begin{array}{l}\text { Pedicelos: } \\
\text { largo (mm) }\end{array}$ & Nulos ó 1 a 2.4 & 2.4 a 4.8 & 2 a 4 & 2.4 a 4.8 \\
\hline $\begin{array}{l}\text { Sépalos: } \\
\text { forma } \\
\text { largo }(\mathrm{mm}) \\
\text { ancho }(\mathrm{mm})\end{array}$ & $\begin{array}{l}\text { linear-oblonga } \\
\begin{array}{l}5 \text { a } 13 \\
2 \text { a } 3.5\end{array}\end{array}$ & $\begin{array}{l}\text { oblongo-elíptica, } \\
\text { lanceolada u ovada } \\
2 \text { a } 8 \\
1.4 \text { a } 1.8\end{array}$ & $\begin{array}{l}\text { lanceolada } \\
2.5 \text { a } 3.5 \\
1 \text { a } 1.5\end{array}$ & $\begin{array}{l}\text { lanceolada a oblon- } \\
\text { ga } \\
3 \text { a } 5 \\
i ?\end{array}$ \\
\hline $\begin{array}{l}\text { Pétalos: } \\
\text { color } \\
\text { forma } \\
\text { largo }(\mathrm{mm})\end{array}$ & $\begin{array}{l}\text { blanco o blanco- } \\
\text { rosado a verde- } \\
\text { blanquecino } \\
\text { oblongo-elíptica } \\
8 \text { a } 11\end{array}$ & $\begin{array}{l}\text { blanco } \\
\text { lanceolada } \\
5.9-7.9\end{array}$ & $\begin{array}{l}\text { blanco } \\
\text { lanceolada } \\
4 \text { a } 5\end{array}$ & $\begin{array}{l}\text { blanco a blanco- } \\
\text { rosado } \\
\text { lanceolada } \\
5 \text { a } 8\end{array}$ \\
\hline $\begin{array}{l}\text { Nectarios: } \\
\text { color }\end{array}$ & rojo oscuro & amarillo pálido & blanco-crema & rojo oscuro \\
\hline $\begin{array}{l}\text { Fenología } \\
\text { (floración) }\end{array}$ & septiembre a febrero & febrero a marzo & julio & octubre a marzo \\
\hline $\begin{array}{l}\text { Altitud a la que } \\
\text { se ha registrado } \\
\text { (m s.n.m.) }\end{array}$ & 2650 a 3250 & 2350 & 1770 & 2100 a 3400 \\
\hline
\end{tabular}




\section{AGRADECIMIENTOS}

Agradecemos sinceramente al Dr. Jerzy Rzedowski la traducción de la diagnosis al latín, a él mismo, a la Maestra Graciela Calderón de Rzedowski, a los Drs. Sergio Zamudio Ruiz y Victor Steinmann la lectura crítica al manuscrito. Al M. en C. Miguel Cházaro, al Ing. J. Antonio Machuca, al Biól. Raúl Acevedo y al Sr. Germán Hernández les agradecemos su apoyo en el trabajo de campo. La ilustración se debe a la pluma del Sr. Rogelio Cárdenas Soriano.

\section{LITERATURA CITADA}

Clausen, R. T. 1959. Sedum of the Trans-Mexican Volcanic Belt: An exposition of taxonomic methods. Cornell Univ. Press. Ithaca, Nueva York. 380 pp.

Clausen R. T. 1978. Sedum- Seven Mexican perennial species. Bull. Torrey Bot. Club 105(3): 214-223. 